\title{
23. FUENTES DEL DERECHO Y REFORMA DE LA
}

CONSTITUCIÓN

FRANCISCO BALAGUER CALLEJÓN

Catedrático de Derecho Constitucional

BALDOMERO OLIVER LEÓN

y

JOSÉ ANTONIO MONTILLA MARTOS

Universidad de Granada 


\section{SUMARIO}

I. LA REFoRMA CONSTITUCIONAL EN MATERIA DE FUENTES. LA CONSTITUCIÓN COMO DERECHO VIVO.-II. LAS FACULTADES DEL JUEZ RESPECTO DE LAS NORMAS LEGALES EN LOS CONFLICTOS ENTRE ORDENAMIENTOS.III. LA ARMONIZACIÓN ENTRE EL SISTEMA DE FUENTES Y LA FORMA DE ESTADO Y DE GOBIERNO.-IV. LA DEFINICIÓN DE LAS POTESTADES NORMATIVAS DE LOS PODERES PÚBLICOS.-V. LA DEFINICIÓN DE LOS CRITERIOS DE ESTRUCTURACIÓN DEL SISTEMA DE FUENTES. 


\section{FUENTES DEL DERECHO Y REFORMA DE LA CONSTITUCIÓN}

POR

FRANCISCO BALAGUER CALLEJÓN

Catedrático de Derecho Constitucional

BALDOMERO OLIVER LEÓN

Y

JOSÉ ANTONIO MONTILLA MARTOS

Universidad de Granada

\section{LA REFORMA CONSTITUCIONAL EN MATERIA DE FUENTES. LA CONSTITUCIÓN COMO DERECHO VIVO}

Una cuestión previa a la que procede hacer referencia, porque afecta además a la consideración de la Constitución como fuente del Derecho, es la del sentido de la reforma constitucional en materia de fuentes. La Constitución no es, como tampoco lo es ninguna otra norma jurídica, el mero texto escrito que se incorporó al ordenamiento jurídico en diciembre de 1978. Más allá de ese texto, la Constitución viva, la Constitución real, es el conjunto normativo que ha surgido del mismo, mediante la interpretación y la aplicación de sus preceptos realizada por los diversos aplicadores del Derecho.

Es importante tener en cuenta esta perspectiva a la hora de definir cuáles son las disposiciones en las que la reforma constitucional en materia de fuentes se impone, y cuáles, por el contrario, no requieren de modificación alguna porque han encontrado acomodo en el ordenamiento 
jurídico sin estridencias que justifiquen una acción normativa de reforma. Partiendo de esa distinción entre el texto constitucional y la Constitución viva, se propugna aqui una autolimitación del poder reformar aquellos aspectos en que resulta estrictamente necesaria su intervención porque los problemas no pueden resolverse por vía de interpretación.

En aquellos supuestos en los que las normas constitucionales vivas no plantean problemas al funcionamiento armónico del ordenamiento, las deficiencias que esas disposiciones constitucionales pudieran tener en su origen quedan aminoradas por la forma en que se ha producido el desarrollo constitucional. Por el contrario, existen ámbitos en los que la Constitución puede requerir modificaciones importantes para hacer frente a las dificultades que encuentra hoy nuestro sistema de fuentes. A este respecto podría decirse que los ámbitos más necesitados de reforma son aquellos que afectan a los problemas estructurales del sistema de fuentes, aquellos que contienen deficiencias estructurales que condicionan el funcionamiento de ese sistema. Avanzando más, podríamos decir que las posibles reformas no son tanto de modificación de preceptos constitucionales, cuanto de incorporación de preceptos que serían necesarios para clarificar las principales interrogantes a que nuestro ordenamiento se abre actualmente. Veamos cuáles son esas interrogantes.

\section{LAS FACULTADES DEL JUEZ RESPECTO DE LAS NORMAS LEGALES EN LOS CONFLICTOS ENTRE ORDENAMIENTOS}

Es este un aspecto que requiere una regulación precisa, cada vez más urgente, desde nuestra incorporación a las Comunidades Europeas. Ya se hizo sentir esa necesidad con la aprobación de los Estatutos de Autonomía y la configuración del Estado constitucional como Estado autonómico. El juez tiene capacidad para discernir, por sí mismo, cuándo debe aplicar preferentemente el derecho estatal o el autonómico infralegal, pero carece de esa capacidad cuando se trata de normas legales. Sin embargo, las colisiones entre las leyes estatales y las autonómicas, por un lado, y entre las leyes internas y los tratados internacionales, y especialmente el Derecho derivado de las Comunidades Europeas, serán, previsiblemente, cada vez más frecuentes. Un sistema de fuentes bien ordenado exige la determinación de los poderes del juez para resolver estos conflictos.

Es obvio, sin embargo, que un ordenamiento como el nuestro no puede conferir al juez la capacidad para decidir, con carácter definitivo, sobre la validez de las normas legales posteriores a la Constitución. Esa fun- 
ción corresponde y debe corresponder tan sólo al Tribunal Constitucional. De ahí que la solución que parece más conveniente a este problema es la de precisar el alcance de la regla de prevalencia respecto de los conflictos normativos entre el Derecho estatal y el autonómico y extender la técnica de la prevalencia a la relación entre el Derecho Comunitario y el Derecho interno de rango legal.

Esa misma extensión es procedente para resolver los conflictos que puedan producirse entre los diversos tipos legales. Es el caso de los conflictos entre ley orgánica y ley ordinaria. La reserva de ley orgánica sólo puede alcanzar efectividad si se establece algún mecanismo por el que la ley orgánica sea aplicada preferentemente sobre la ordinaría, en caso de conflicto, hasta que, en su caso, decida el Tribunal Constitucional sobre los límites materiales que afectan a uno y otro tipo legal.

La técnica de la prevalencia alcanza aqui su mayor virtualidad, como instrumento destinado a superar al criterio jerárquico en todos aquellos supuestos en los que la solución de los conflictos normativos requiera algún tipo de delimitación competencial. El instituto de la prevalencia resulta más congruente con el sistema de fuentes propio de un ordenamiento complejo que el de la jerarquía. $Y$ ello porque el criterio jerárquico es, en realidad, la manifestación más genuina de un sistema de fuentes simple ordenado en torno a la ley como fuente suprema del ordenamiento. El criterio jerárquico se basa en una relación internormativa directa que sólo se puede predicar hoy para aquellos ámbitos del sistema de fuentes en los que la relación entre normas no esté mediatizada por criterios competenciales, esto es, por una tercera norma que defina los límites de cada uno de los poderes normadores. El supuesto típico de aplicación del criterio jerárquico es la relación entre ley y reglamento en el interior de cada ordenamiento.

La aplicación de la técnica de la prevalencia a estos supuestos de relaciones entre ordenamientos o entre tipos normativos dentro de una misma fuente, permitirá resolver los conflictos normativos mediante el desplazamiento (provisional) de una de las normas y la aplicación subsiguiente de la otra. En el caso de la relación entre ley orgánica y ley ordinaria se aplicará preferentemente la ley orgánica (o en el de la ley de armonización y las leyes autonómicas, la ley de armonización). En el caso de la relación entre le Derecho estatal y el autonómico, se aplicará preferentemente el Derecho estatal, salvo en los supuestos de competencias exclusivas de la Comunidad Autónoma. En la relación entre el Derecho Comunitario y el Derecho interno, se aplicará el Derecho Comunitario. Todo ello, naturalmente, siempre que el juez entienda que cada una de las normativas preferentes se ha producido dentro del marco competencial propio. 
El problema, por tanto, se plantea en aquellos supuestos en los que el juez estima que la normativa preferente ha rebasado el ámbito competencial propio (si se trata de Derecho estatal que no afecte a las competencias exclusivas de las CC.AA., pues en ese caso bastaría con aplicar el Derecho autonómico, e igualmente si se trata de leyes orgánicas que han regulado materia no sujeta a reserva específica), o ha incurrido en incompatibilidad con la Constitución (si se trata de Derecho comunitario). La única solución actual al problema estriba en promover ante el TC la cuestión de inconstitucionalidad, para que sea este órgano el que resuelva. Solución que si bien parece necesario mantener, no es satisfactoria para resolver algunos de estos conflictos normativos y que, además, no está al alcance de otros aplicadores del Derecho.

La única solución razonable a estos supuestos es la de la aplicación, en todo caso, del derecho preferente lo preferente en su ámbito, como es el caso del Derecho estatal frente al Derecho autonómico que no se produce en el ejercicio de las competencias exclusivas de las CC.AA.). Ello implica que todo aplicador del Derecho deberá optar por esa normativa con carácter preferente. Tan sólo el juez ordinario podrá valorar, en su caso, si las circunstancias aconsejan el planteamiento o no de la cuestión de inconstitucionalidad, ponderando todos los intereses en juego.

Todo lo anterior nos lleva a proponer ya en este punto una posible reforma cuya sede lógica parece ser el art. $163 \mathrm{CE}$, a cuya redacción actual se añadiria un segundo párrafo del siguiente tenor:

163:

“... No será necesario plantear la cuestión de inconstitucionalidad en aquellos supuestos en los que la norma de rango legal sea contraria al Derecho comunitario o a los Tratados Internacionales que formen parte del ordenamiento interno. Tampoco será necesario plantearla en los supuestos en que se produzca una colisión normativa entre el Derecho estatal y el autonómico o entre una ley ordinaria y una ley orgánica $u$ otros tipos normativos específicos".

Igualmente, sería necesario añadir algún otro párrafo a otros preceptos constitucionales:

96.3:

«El Derecho internacional vigente en España y el Derecho comunitario prevalecerán sobre el Derecho interno». 
81.3:

"Las leyes orgánicas prevalecerán sobre las ordinarias dentro de la reserva material propia".

150.3:

"... Las leyes de armonización prevalecerán sobre cualquier otra ley estatal o autonómican.

\section{LA ARMONIZACIÓN ENTRE EL SISTEMA DE FUENTES $Y$ LA FORMA DE ESTADO Y DE GOBIERNO}

Es éste un punto en el que también se puede constatar, cuando menos, un claro desfase entre el texto de la Constitución en materia de forma de gobierno y en lo referente al sistema de fuentes. Un régimen parlamentario como el nuestro, basado en la diferenciación entre mayoría-oposición, exige un sistema de fuentes que responda a los criterios de estructuración de la forma de gobierno. Ello supone que no tiene sentido hoy propugnar la exclusividad de las Cortes Generales sobre la función legislativa. El Gobierno (que representa sólo a la mayoría parlamentaria) ejercita una función legislativa importante, ya sea de urgencia o delegada.

Esa función legislativa gubernamental parece hoy absolutamente necesaria. Es claro sin embargo, que en este punto, nuestra Constitución padece un cierto desajuste interno que podría explicarse por el desfase que entre la realidad y las normas se produce cuando las normas son el resultado de la implantación de una determinada mentalidad que se construye en relación con realidades ya superadas. Es lo que ocurre con algunos preceptos constitucionales que son el resultado claro del rechazo al régimen franquista, y más aún, el resultado de la lucha por democratizar el régimen franquista, pero que no resultan operativos para un sistema democrático basado en un sistema propio que no se puede construir desde el rechazo al anterior, sino desde su propia lógica.

Algunas de las restricciones constitucionales a la potestad legislativa del Gobierno, se mueven dentro de este contexto. La exigencia de una situación de "extraordinaria y urgente necesidad" para que el Gobierno pueda dictar Decretos-Leyes, es un caso claro. Cierto es, sin embargo, que el presupuesto habilitante de la legislación "de urgencia" ha sido interpre- 
tado extensivamente por el TC, y en ese sentido la reforma constitucional no aparece como una exigencia inexcusable.

Lo mismo se puede decir de algunas limitaciones a la legislación delegada o de la consideración mecanicista de la actividad del Gobierno en la refundición de textos legales, que, según el art. 82.5 CE puede reducirse "a la mera formulación de un texto único" sin intervención normativa alguna del Gobierno.

Igualmente criticable es, a estos efectos, la concepción todavía dominante de los Decretos legislativos como fruto del ejercicio de una potestad reglamentaria que puede ser controlada por los tribunales ordinarios. No existe justificación alguna, en el sistema de fuentes constitucional, para el control jurisdiccional ordinario de los excesos competenciales de normas con rango de ley. Es cierto, sin embargo, que los tribunales pueden intervenir en los procesos de aplicación de normas legales, decidiendo sobre su vigencia. También lo es que aquí se ha defendido una extensión de las facultades del juez respecto de la aplicación de normas legales en los supuestos de conflictos entre ordenamientos. Pero la decisión sobre la validez de las normas legales debe corresponder al Tribunal Constitucional.

En este punto sí parece necesaria una reforma del texto constitucional que clarifique la situación. Esa reforma podría ser del siguiente tenor:

\section{6:}

"Las leyes de delegación podrán establecer fórmulas de control de la legislación delegada».

Para completar los apuntes aportados en este epígrafe es necesario referirse, aun cuando no podamos desarrollar en toda su extensión el tema, al papel que nuestra Constitución reconoce al Rey en relación con el sistema de fuentes.

En líneas generales, la primera cuestión que conviene resaltar es que, debido fundamentalmente a la irresponsabilidad e inviolabilidad del Monarca, el carácter normativo de la Constitución no ha desplegado toda su virtualidad en relación con el Título II de la misma, ya que, aun cuando doctrinalmente es ampliamente aceptado que las funciones que la Constitución concede al Rey tienen la consideración de actos debidos para el Monarca, del incumplimiento o de un cumplimiento inapropiado o abusivo de dichas funciones por parte de su titular, precisamente por su irrespon- 
sabilidad e inviolabilidad, no se derivan consecuencia jurídica alguna. Por tanto, la armonización de la posición constitucional del Rey con la eficacia en el desenvolvimiento del sistema de fuentes (y en general con el desarrollo de nuestro sistema político-constitucional) requiere la insistencia en la consideración como actos debidos de las funciones que constitucionalmente se le otorgan al Rey.

Aceptada esta premisa, así como el carácter estrictamente formal y simbólico de las funciones del Rey, su participación en la implementación del sistema de fuentes (esencialmente por lo que se refiere a la sanción y promulgación de las leyes y a la expedición de los decretos acordados en el Consejo de Ministros) supone en la redacción actual del texto constitucional, un requisito para la formalización y estatalización de la actividad de los diferentes órganos de producción jurídica. Sin embargo, esa condición se explica tan sólo por la dimensión simbólica del Rey y por el peso de la densa tradición monárquica. Su mantenimiento en el texto constitucional podría llegar a resultar disfuncional sin que, por contra, esta participación regia en el desarrollo del sistema de fuentes aporte ningún elemento sustancial para la manifestación de la voluntad normativa de los distintos órganos de producción jurídica.

\section{LA DEFINICIÓN DE LAS POTESTADES NORMATIVAS DE LOS PODERES PÚBLICOS}

No existe en la Constitución un concepto claro de lo que es y lo que no es normativo. No es esa, por otra parte, una función propia de la Constitución. Sin embargo, este problema se torna más complejo en la medida en que se cruza con el de la definición del ámbito de poder "normativo" de los diversos poderes públicos, y especialmente del Parlamento y del Gobierno.

La diferente consideración que para nuestra doctrina y nuesta jurisprudencia ofrece el poder reglamentario frente al poder legislativo, se manifiesta especialmente en la exigencia de requisitos específicos para el ejercicio de un poder reglamentario que presente una determinada estructura formal. Es el caso de la definición de lo básico por medio de Reglamentos en la distinción entre legislación básica y legislación de desarrollo. Es el caso, igualmente, de la posibilidad, que no se ofrece al reglamento, de producir leyes formales de carácter singular o para un caso único. La distinción entre reglamento y acto administrativo, que no tiene equivalencia en nuestro ordenamiento para actos aprobados formalmente como 
leyes por el Parlamento (que se consideran leyes cualquiera que sea su contenido), es una muestra de la distinta consideración que puede tener un mismo acto, como normativo o como no normativo, en función de un criterio formal como es la procedencia de un determinado órgano.

Es cierto, sin embargo, que existen otros criterios adicionales que pueden justificar una diferente consideración del mismo acto como normativo o como no normativo. En el caso de la ley singular, la condición derogatoria del acto respecto a la legislación previa del Parlamento, le confiere cualidad normativa a ese acto. Esto no ocurre en la distinción entre reglamento $y$ acto administrativo debido al principio de inderogabilidad singular de los reglamentos.

La cuestión es si debe existir un pronunciamiento expreso de la Constitución que clarifique las condiciones en que debe ejercerse el poder público para que pueda ser considerado como normativo. A este respecto no parece que los problemas que se han planteado hasta ahora tengan que resolverse necesariamente a nivel constitucional (si es que ello es posible, por otra parte). Más bien, se trata, de momento, de un problema de evolución doctrinal y jurisprudencial. De hecho, si tenemos que optar por un criterio sustancial o procedimental de la normatividad, la única elección adecuada parece ser la segunda, pues la opción por un criterio sustancial implicaría una contradicción con los preceptos constitucionales en los que se prevé la existencia de leyes de contenido no normativo en sentido sustancial.

\section{LA DEFINICIÓN DE LOS CRITERIOS DE ESTRUCTURACIÓN DEL SISTEMA DE FUENTES}

La cuestión de los criterios de estructuración del sistema de fuentes está estrechamente conectada con la de los órganos que deben tener capacidad para fiscalizar la validez-legitimidad de las distintas normas respecto del ordenamiento jurídico. Así, sólo es posible hablar de jerarquía cuando existe un mandato a los aplicadores del Derecho para que, en caso de conflicto normativo, determinen por sí mismos la ilegitimidad de la norma inferior. Del mismo modo, la prevalencia es un mandato de inaplicación de determinadas normas que no resuelve respecto de la validez de esas normas.

Sin embargo, la conexión que se da entre ambos fenómenos, no debe llevarnos a la confusión de los mismos. Esta confusión puede produ- 
cirse si no tenemos en cuenta que uno de estos criterios, el de competencia, por tratarse de una apelación específica a la validez-legitimidad de las normas, cuando afecta a normas legislativas puede requerir de la definición previa de un parámetro normativo que debe servir de criterio de legitimidad de las normas enjuiciadas (cuestión ésta que no es necesaria cuando interviene el criterio de jerarquía, ya que entonces se produce una relación internormativa directa en la que la simple contradicción de la norma inferior con la superior determina su invalidez). La definición de ese parámetro se produce en el momento de la actividad jurisdiccional y no se basa en una relación aislada entre normas, sino en la obligación de todos los poderes normativos (de todas las normas, por tanto) de respetar el ordenamiento jurídico. Respetar el ordenamiento jurídico significa, ante todo, respetar la actuación de cada poder normativo en el ámbito de sus competencias. Ello supone que las normas que están condicionadas por el parámetro de constitucionalidad, pueden a su vez, ser condicionantes de otras normas e integrar ese mismo parámetro para otras normas, porque no se trata de una relación jerárquica la que se establece aquí, sino de una relación competencial entre normas. Por tanto, entre las normas que sirven de parámetro y las controladas, no existe relación jerárquica.

La cuestión es si resulta o no necesario que en la propia Constitución se definan con detalle todas estas cuestiones, y por tanto, los criterios de estructuración del ordenamiento en su conjunto. Ante todo, no es imputable estrictamente a la Constitución una interpretación en clave jerárquica del ordenamiento. Por el contrario, si esa interpretación existe, es más el producto de una cierta inercia doctrinal que asume la normatividad de la Constitución sin conectarla con sus presupuestos políticos y sociales, $\checkmark$ por tanto, sin incardinarla en el seno del valor pluralismo y de la estructura compleja del ordenamiento que de la propia Constitución se deriva.

Cabe plantearse entonces si es necesario que la Constitución «explique" el sentido de su normatividad mediante la clara definición del significado que debe darse al principio de competencia en todas sus variantes, y específicamente en lo que se refiere a los bloques normativos que deben servir como parámetros para el control de constitucionalidad de otras normas (el caso del bloque de constitucionalidad). En mi opinión, eso no es necesario, y podría producir una cierta rigidez en ámbitos del ordenamiento en los que la flexibilidad es más necesaria, pues en ellos el-nuevo ordenamiento que surge de la Constitución normativa está todavía en fase de "construcción". 